\title{
Sensilla on the Adult and Larval Antennae of Cotinis nitida (Coleoptera: Scarabaeidae)
}

\author{
G. T. Baker, * W. A. Monroe** \\ * Entomology \& Plant Pathology, Box 9775, Mississippi State University, Mississippi State, MS \\ 39762, ** EM Center, Box 9775, Mississippi State University, Mississippi State, MS 39762
}

Turfgrass production and management is a billion dollar industry in the United States and Canada. Besides using proper agronomic practices to ensure healthy turfgrasses, weeds, pathogens and insect pests must also be managed in an economically efficient and an environmentally safe manner. One of the major groups of the many insect pests are the white grubs, which are the larvae of beetles from the family Scarabaeidae. [1]. White grubs are distributed over a very large area and feed on many cultivars from several important turfgrass species. Insects have a wide variety of cuticular sensory receptors on their antennae, mouthparts, legs, ovipositor and other body regions that are involved in many olfactory and gustatory functions $[2,3]$. Cuticular sensilla are known to perceive many chemical compounds that control many intraspecific and interspecific behavior of insects [4, 5]. Before electrophysiological and behavioral studies are initiated, the morphology, distribution and number of cuticular sensilla on the antennae of Cotinis nitida need to be established.

For transmission electron microscopy (TEM) adults and larvae of Cotinis nitida were fixed in halfstrength Karnovsky's fixative in phosphate buffer at $\mathrm{pH} 7.2$, overnight at $4^{\circ} \mathrm{C}$ and then post-fixed in $2 \%$ osmium tetroxide in the same buffer for $2 \mathrm{~h}$ at room temperature. Specimens were dehydrated in a graded series of ethanol, and then infiltrated slowly and embedded in Spurr's low viscosity embedding medium [6]. Thin sections were stained with uranyl acetate and lead citrate and were examined with a JEOL CX 100 II TEM at $60 \mathrm{kV}$. The same fixation and dehydration procedures were used to prepare specimens for scanning electron microscopy (SEM). The specimens were then dried with HMDS (hexamethyldisilazane), mounted on aluminum stubs with carbon tape, sputtercoated with gold-palladium, and examined with a LEO $360 \mathrm{~S} \mathrm{SEM}$ at $15 \mathrm{kV}$. Adults and larvae were stained with silver nitrate in order to test for the porosity of the various cuticular sensilla on the antennae [7].

The three apical antennomeres of the adult antenna form a lamellate, club-like structure (Fig. 1). The cuticular sensilla are situated on the inner surface of lamellae I and II and on both the ventral and dorsal surfaces of lamella II (Fig. 2). There are three types of sensilla; (1) sensillum placodeum varies in diameter (5.5 um - $12.0 \mathrm{um}$ ) and are irregularly shaped (Fig 3); stained intensely with silver nitrate indicating a porous cuticle; two sensory neurons are associated with the plate sensilla. (2) sensillum basiconicum is deeply grooved and 4.0 - 4.5 um long and $2.2-2.4$ um wide at the base; stained very black by the silver nitrate indicating a porous cuticle; three sensory neurons are associated with the sensillum; each basiconic sensillum is in a shallow depression about $5.0-7.0 \mathrm{um}$ wide about 3.0 - 35 um deep. (3) sensillum coeloconicum are smooth and 1.8 - 2.3 um long and 1.4 - 1.6 um wide at the base; (Fig. 3) located in pits that are $2.3-2.6$ um wide; stain intensely with silver nitrate; with two sensory neurons. The larval antenna has four antennomeres and is devoid of setae (Fig. 4). The two types of sensilla that are found on the larval antenna are concentrated on the apical antennomere. There are eight peg sensilla on the apex which are 7.5-8.5 um long and $3.5-$ $4.0 \mathrm{um}$ wide at the base (Fig. 5) and stain with silver nitrate. Two sensory neurons are found with 
the peg sensillum. Sensilla placodea are situated on the dorsal and ventral surfaces of the apical antennomere (Fig. 6) and are much wider in diameter than those found on the adult, 30.0 - $90.0 \mathrm{um}$ wide. As with the adult sensilla placodea, the larval ones stain with silver nitrate and have two sensory neurons.

[1] P. J. Vittum et al., Turfgrass Insects of the United States and Canada, $2^{\text {nd }}$ ed., Cornell University Press, Ithaca, 1999.

[2] R. F. Chapman, Insects: Structure and Fucntion, $2^{\text {nd }}$ ed., Cambridge University Press,

Cambridge, 1998.

[3] R. Y. Zacharuk, Ann. Rev. Entomol. 25 (1980) 27.

[4] T. L. Payne et al., Mechanisms in Insect Olfaction, Clarendon Press, Oxford, 1986.

[5] E. Hartliek and P. Anderson, in Insect Olfaction, B. S. Hansson (ed), Springer, Berlin, 1999.

[6] G. T. Baker et al., Invertebrate Biol. 119 (2000) 342.

[7] R. Schafer and T. V Sanchez, J. Morphol. 149 (1976) 139.

[8] This was partially supported by NSF Grants DIR-9001495 and DEB-9200856 and the Mississippi Agricultural and Forestry Experiment Station.
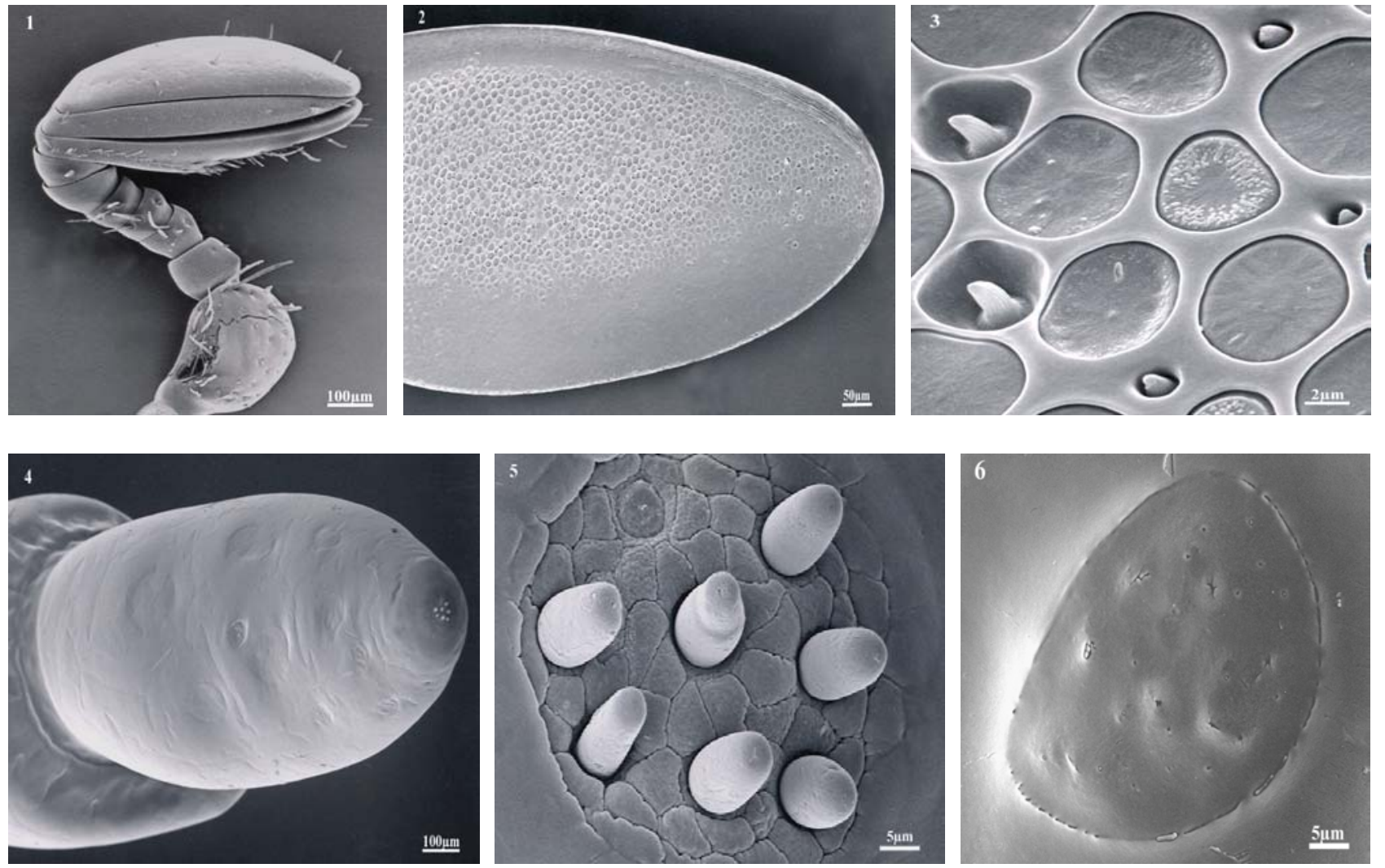

Figs. 1-3 Adult antenna. 1. Lamellae of the antenna. 2. Inner surface of the lamella, 3. Three types of sensilla. Fig. 4-6 larval antenna. 4. Apical antennomere. 5. Peg-like sensilla on the antennal apex. 6. Plate-like sensillum. 